ARTICLE

DOI: $10.1038 / s 41467-018-06534-1$

\title{
Direct electrical quantification of glucose and asparagine from bodily fluids using nanopores
}

Nicole Stéphanie Galenkamp ${ }^{1}$, Misha Soskine ${ }^{1}$, Jos Hermans ${ }^{10}{ }^{2}$, Carsten Wloka ${ }^{1} \&$ Giovanni Maglia ${ }^{1}$

Crucial steps in the miniaturisation of biosensors are the conversion of a biological signal into an electrical current as well as the direct sampling of bodily fluids. Here we show that protein sensors in combination with a nanopore, acting as an electrical transducer, can accurately quantify metabolites in real time directly from nanoliter amounts of blood and other bodily fluids. Incorporation of the nanopore into portable electronic devices will allow developing sensitive, continuous, and non-invasive sensors for metabolites for point-of-care and home diagnostics.

\footnotetext{
${ }^{1}$ Groningen Biomolecular Sciences and Biotechnology Institute, University of Groningen, Nijenborgh 7, Groningen 9747 AG, The Netherlands. ${ }^{2}$ Analytical Biochemistry, Department of Pharmacy, University of Groningen, Antonius Deusinglaan 1, Groningen 9713 AV, The Netherlands. Correspondence and requests for materials should be addressed to G.M. (email: g.maglia@rug.nl)
} 
T he ability to monitor the health of individuals that exchange information through the Internet without requiring human intervention would undoubtedly bring about a revolution in personalised healthcare. Over the past decades, the miniaturisation of electronic devices combined with recent advances in the wireless transmission of data and microfluidics have opened up new possibilities for making such devices. However, one key area that is preventing their breakthrough is the production of sensing elements for biologically relevant molecules, which can be easily interfaced with silicon-based electronics. Such elements have long lagged behind the electronic industry in terms of miniaturisation and cost reduction.

At the moment, biosensors that can be integrated in electronic devices rely mostly on the electric signal that is generated by the oxidation of a target analyte catalysed by an immobilised enzyme $^{1}$. However, such oxidase enzymes are not ubiquitous, and today's commercially available home diagnostic and implantable devices are entirely limited to one well-known example: the glucose sensor. However, despite the sensor has seen many improvements since it was first introduced in the early $1960 \mathrm{~s}^{2}$, it still suffers from limitations including enzyme instability, complicated surface immobilisation procedures, baseline shifts, and does not tolerate well changes in micro-environmental factors such as changes in $\mathrm{pH}$ and ionic strength ${ }^{3-5}$.

Nanopores, which are nanometre-sized water conduits spanning biological or artificial membranes, are emerging as a new class of biosensors. When a potential is applied, the flux of ions across an individual nanopore generates an electrical signal that allows direct interfacing with electronic devices. Most notably, nanopores can be integrated into electronic devices and are now used for low-cost, high-speed, and portable sequencing of $\mathrm{DNA}^{6}$. Nanopores can also detect polypeptide 7,8 biomarkers. However, their use in diagnostic devices is complicated by the low, typically picomolar, concentration of such biomarkers in biological fluids. By contrast, metabolites are highly abundant in bodily fluids, and their variation in concentration is linked to many diseases 9 . Nanopores equipped with cyclodextrins ${ }^{10}$, cucurbiturils ${ }^{11}$, or cyclic peptides ${ }^{12}$ may recognise metabolites. However, the detection and quantification of such analytes directly from a biological sample cannot be easily implemented because the recognition elements lack selectivity.

Recently, we have shown that the biological nanopore cytolysin A (ClyA) can be used to monitor the function of proteins when they are lodged inside the nanopore (Fig. 1a) ${ }^{13-16}$. Here, using proteins from a protein family that in cells recognise an enormous variety of molecules, we show that ClyA nanopores can report the concentration of glucose and asparagine directly from samples of blood, sweat, and other bodily fluids. Conveniently, no sample preparation is required and the concentration of the metabolite can be monitored continuously.

\section{Results}

Characterisation of the glucose-binding protein. In this work, we used a glucose-binding protein (GBP) that binds glucose with high affinity and specificity ${ }^{17}$. GBP belongs to the class of substrate-binding proteins which, with minor structural differences, recognise hundreds of different metabolites in cells ${ }^{18}$. The structure of the GBP has been solved in the ligand-free state (open or apo form) configuration and in the closed, ligandbound, configuration. Interestingly, NMR studies revealed that in solution, apo-GBP adopts both configurations even in the absence of glucose ${ }^{19}$. In our experimental setup, GBP $(44 \mathrm{nM})$ was added to the cis side of a ClyA-AS ${ }^{20}$ nanopore (hereafter ClyA, Fig. 1a). $\mathrm{A}-90 \mathrm{mV}$ voltage bias was then applied across the membrane, which generates an electro-osmotic flow across the nanopore promoting the entry of GBP inside ClyA, as observed by an ionic current blockade (Fig. 1a). Under these conditions, proteins remained trapped inside the nanopore for an average time of 10.1 $\pm 1.7 \mathrm{~s}(-90 \mathrm{mV}, n=308$ events, $N=3$ independent nanopore experiments). Two current levels ( $\mathrm{L} 1, I_{\mathrm{res} \%}=69.1 \pm 0.2 \%$, and L2, $I_{\text {res } \%}=67.7 \pm 0.3 \%$, with $I_{\text {res } \%}$ indicating the percentage ratio between the open pore current and the blocked pore current, $N=5$, Fig. 1b) could be observed within the ionic current blockade of GBP. The relative time spent by the protein on L1 (28\%) and L2 (72\%) corresponded well to the percentage of closed and open configuration measured for apo-GBP by NMR spectroscopy (32 and 68\%, respectively) ${ }^{19}$.

Addition of glucose to the trans side of the nanopore increased the number of events corresponding to the closed configuration (L1, Fig. 1b, c), while the duration of the events remained nearly constant (Fig. 1c). As expected for a binding process, the frequency of blockades scaled linearly with the concentration of the ligand (Fig. 1c). The ligand-induced closing $\left(k_{\mathrm{closing}}=6.7 \pm\right.$ $\left.0.3 \times 10^{6} \mathrm{~s}^{-1} \mathrm{M}^{-1}\right)$ and opening $\left(k_{\text {opening }}=0.75 \pm 0.12 \mathrm{~s}^{-1}\right.$, $500 \mathrm{nM}$ glucose) rate constants could be determined from the inverse of L1 and L2 dwell times (Fig. 1c), respectively, and were similar to the values measured by stopped flow $\left(k_{\text {closing }}=3-4 \times\right.$ $10^{7} \mathrm{~s}^{-1} \mathrm{M}^{-1}$ and $\left.k_{\text {opening }}=1.4 \mathrm{~s}^{-1}\right)^{21}$. The dependence of the fractional time of GBP in the closed configuration with the glucose concentration fitted well to a Hill function with the Hill coefficient set to one (Supplementary Fig. 1a), from which a normalised calibration curve could be obtained (Fig. 1c). The extrapolated apparent binding constant $\left(2.3 \pm 0.1 \times 10^{-7} \mathrm{M}\right)$ corresponded well to the value measured in bulk $\left(2-5 \times 10^{-7}\right.$ $M)^{21-23}$. A GBP variant (GBP-A213R) with reduced affinity for glucose $^{24,25}$ showed only one single level in the absence of glucose $\left(I_{\text {res\% }}=67.7 \pm 0.1 \%, \quad k_{\text {closing }}=4.69 \pm 0.42 \times 10^{6} \mathrm{~s}^{-1} \mathrm{M}^{-1}\right.$ and $k_{\text {opening }}=121.0 \pm 6.9 \mathrm{~s}^{-1}$, Supplementary Fig. 2), corresponding to the open configuration of GBP. GBP-A213R closed at higher concentrations of glucose $(20 \mu \mathrm{M}$, Supplementary Fig. 2). Together, these results indicate that nanopore currents can report the binding of glucose to GBP, and that the concentration of glucose inside the nanopore is similar to the concentration of glucose in bulk.

Quantification of glucose from human biological fluids. Next, we measured the concentration of glucose directly from a panel of biological samples. Aliquots of blood $(10 \mathrm{~nL})$, sweat $(2 \mu \mathrm{L})$, urine $(200 \mathrm{~nL})$, and saliva $(8 \mu \mathrm{L})$ were added to the trans chamber of an electrophysiology setup containing a single ClyA nanopore. The untreated samples did not alter the stability of the lipid bilayer over the experimental timescale (up to $100 \mathrm{~min}$, Supplementary Fig. 3). Large molecules could not enter the narrow trans side of the nanopore, while small molecule such as glucose could freely diffuse and bind to the trapped GBP previously added to the cis chamber $(44 \mathrm{nM})$. Extrapolation from the calibration curve (Fig. 1c) allowed measuring the concentration of glucose directly from the diluted biological samples. Comparison with values measured with existing commercial glucose test kits showed that the nanopore accurately measured the concentration of glucose in those biological samples (Table 1 and Supplementary Table 1).

Detection of asparagine. One of the advantages of the nanopore technique is that single molecules are detected, which in turn allows the measurement of multiple analytes. To explore this concept, we used SBD1, a substrate-binding domain analogous to GBP that recognises asparagine rather than glucose. Asparagine is a marker for brain damage in acute stroke ${ }^{26}$, while its abnormal concentration in blood has been associated to Parkinson's disease $^{27}$. Under $-90 \mathrm{mV}$, the blocked pore level of apo-SBD1 
a

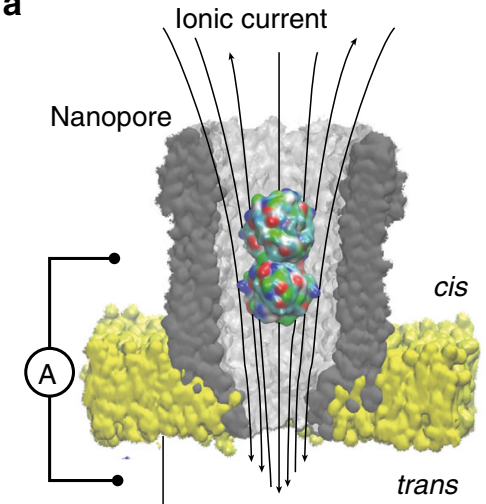

Lipid bilayer
Glucose binding protein

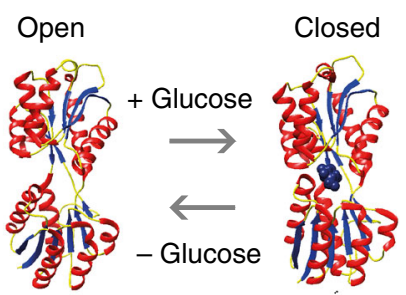

Current (pA)

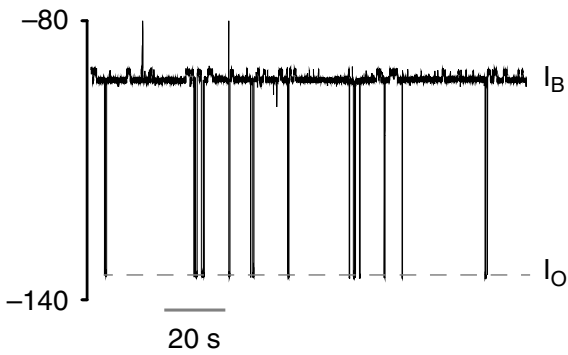

c

Counts $\left(\times 10^{4}\right) \quad$ open
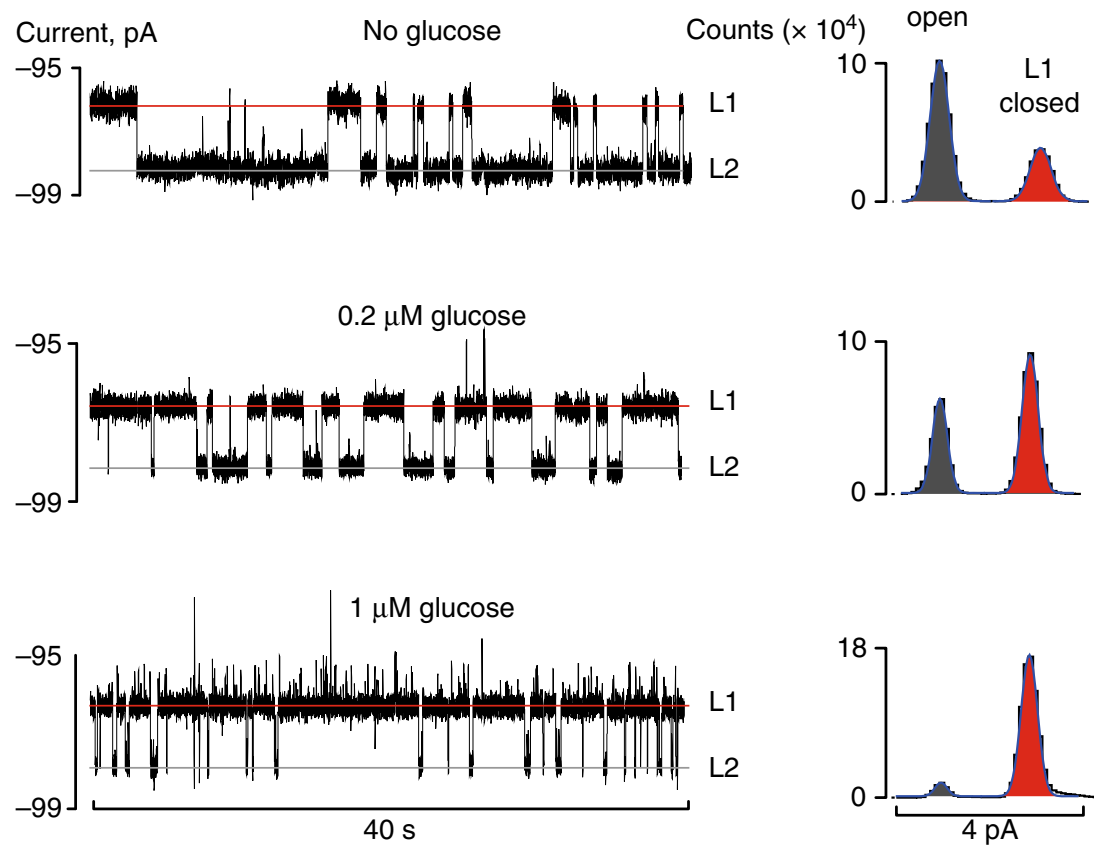

Rate, $\mathrm{s}^{-1}$

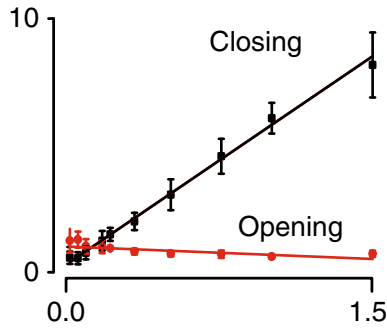

[Glucose], $\mu \mathrm{M}$

Bound fraction

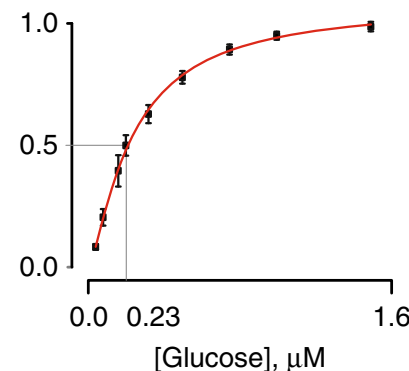

Fig. 1 Nanopore glucose sensor for biological samples. a Cut-through of a surface depiction of a ClyA nanopore (grey) inserted into a lipid bilayer (yellow) containing a glucose-binding protein (GBP) created with the VMD software. GBP residues are coloured by the software according to their type. On the right are cartoon representations of GBP proteins in the open, ligand-free state and closed, liganded state. Right from the cartoon is a typical output signal (current trace) showing the entry of GBP proteins inside the nanopore. $I_{B}$ is the blocked pore current signal and $I_{O}$ the open pore current signal. $\mathbf{b}$ Effect of increasing concentrations of glucose added to the trans solution to the signal induced by GBP proteins internalised into a ClyA nanopore at $-90 \mathrm{mV}$. On the left are shown current traces, on the right all-point histograms of $\sim 50 \mathrm{~s}$ of a current trace. c Dependency of the opening and closing rates, measured as the inverse of dwell times (top) and the fraction of the closed state (bottom) on the concentration of glucose in the trans solution. The lines in the top graph are linear fits and the line in the graph below indicates the fitting to a Hill function. The solution used for the electrical recordings contained $150 \mathrm{mM} \mathrm{NaCl}$ and $15 \mathrm{mM}$ Tris- $\mathrm{HCl}$ set at $\mathrm{pH}$ 7.5. Current traces were collected applying a Bessel low-pass filter with a $2 \mathrm{kHz}$ cutoff and sampled at $10 \mathrm{kHz}$ at room temperature $\left(25^{\circ} \mathrm{C}\right)$. A post-acquisition Gaussian filter of $200 \mathrm{~Hz}$ was applied. Error bars represent the standard deviation between independent experiments $(N=3)$

contained one main current level corresponding to the open protein configuration (Fig. 2a). The addition of asparagine to the trans side of the nanopore led to the appearance of an additional current level corresponding to the closed configuration of SBD1 (Fig. 2a), from which binding constants could be measured (Fig. $2 \mathrm{~b}, k_{\text {off }}=8.6 \pm 0.1 \mathrm{~s}^{-1}, k_{\text {on }}=1.8 \pm 0.1 \times 10^{7} \mathrm{M}^{-1} \mathrm{~s}^{-1}, K_{\mathrm{d}}$ app $=0.50 \pm 0.04 \mu \mathrm{M})$. As observed before for GBP, the binding constants measured by the nanopore were similar to the ones measured in bulk ${ }^{16,28}$. The percentage of bound fraction was used to extrapolate the concentration of asparagine in the trans solution (Fig. 2c). Comparison with an established technique, high performance liquid chromatography (HPLC) coupled with fluorescence detection indicated that the nanopore approach can accurately quantify the concentration of asparagine in all biological samples (Table 1, Supplementary Fig. 4, and Supplementary Table 1). Measurements of asparagine in the presence of high concentration of glucose, glutamine, and glycine (Supplementary Fig. 5) confirmed that SBD1 shows no appreciable off-target binding activity. Measurements of asparagine in urine with known increasing amounts of asparagine (standard additions) indicated that there is no matrix effect (Supplementary Fig. 6). 
Table 1 Comparison of different methods measuring the concentrations of glucose and asparagine in different biological samples

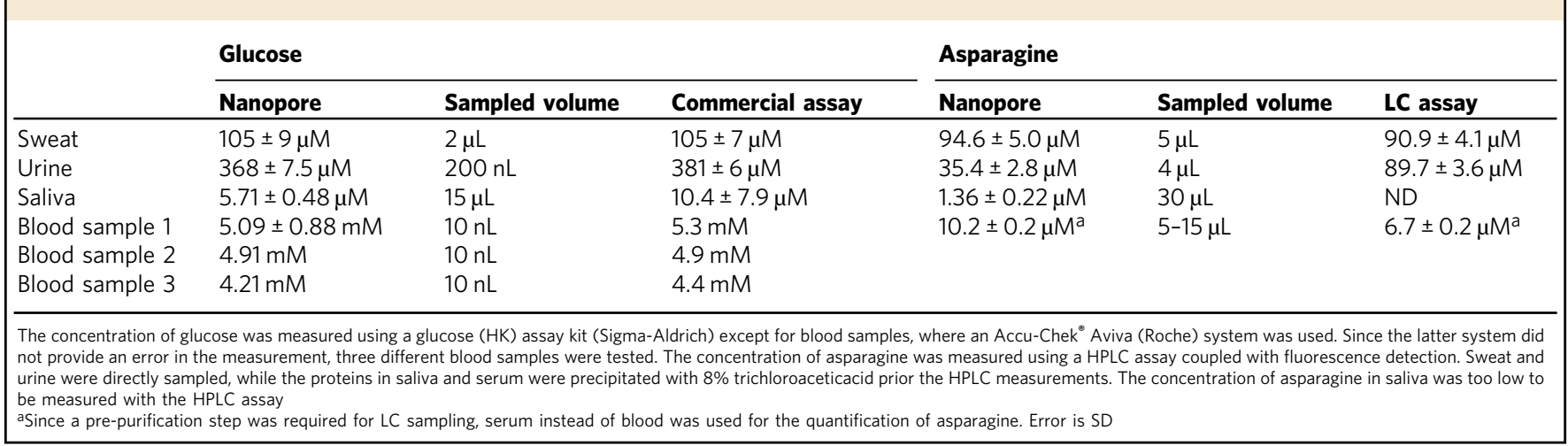

a
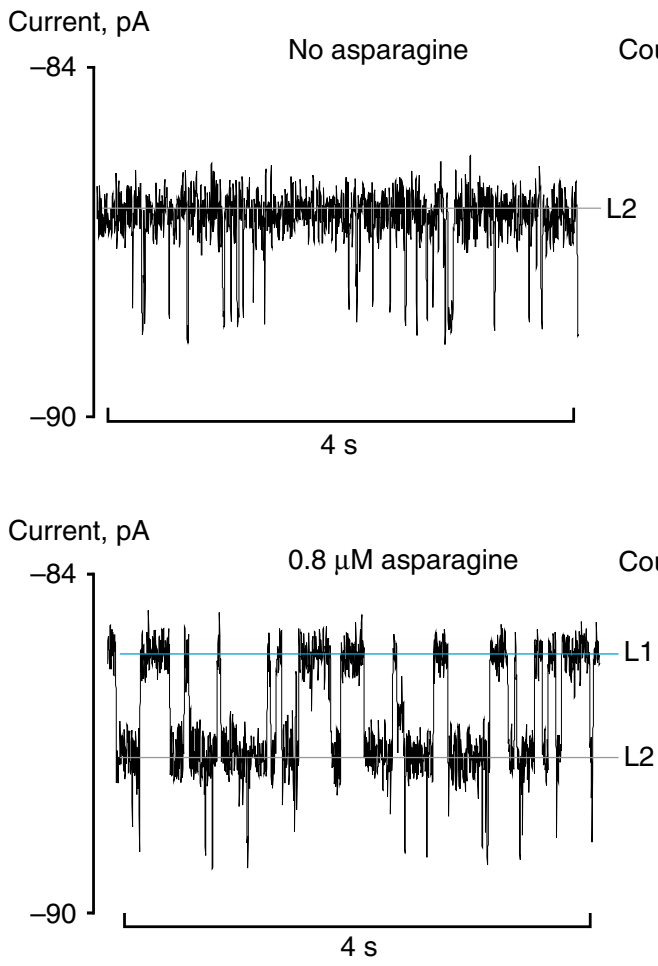

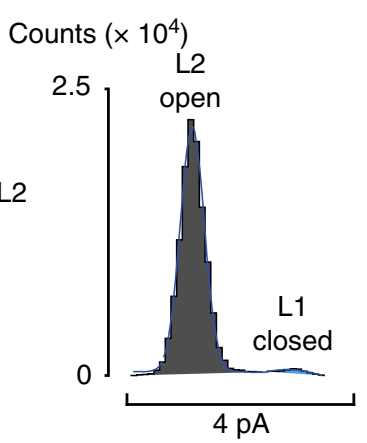

Counts $\left(\times 10^{4}\right)$

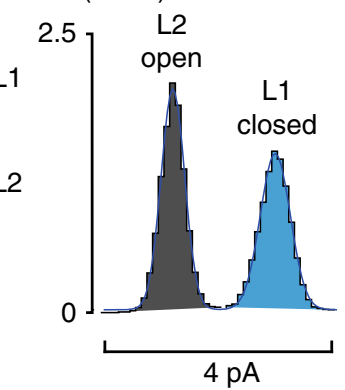

b

Rate, $\mathrm{s}^{-1}$

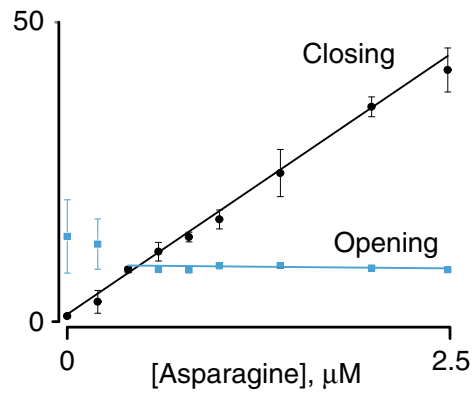

C

Bound fraction

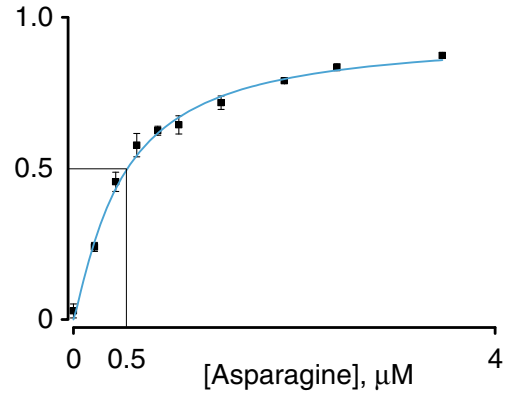

Fig. 2 Binding constants for asparagine binding to SBD1. a Current traces and all-point current histograms showing the binding of asparagine to SBD1 (120 $\mathrm{nM}$, cis) inside a ClyA nanopore. Asparagine was added to the trans solution and the voltage was set to $-90 \mathrm{mV}$. $\mathbf{b}$ Dependency of the opening and closing rates (calculated from the inverse of the dwell times) on the concentration of asparagine in the trans solution. The black and blue lines are linear fits. c Dependency of the percentage of the closed configuration on the concentration of asparagine in the trans solution. The blue line indicates fitting to a Hill function with the Hill coefficient set to one. The solution used for the electrical recording contained $150 \mathrm{mM} \mathrm{NaCl}, 15 \mathrm{mM} \mathrm{Tris-HCl,} \mathrm{pH} \mathrm{7.5.} \mathrm{Current} \mathrm{traces}$ were collected applying a Bessel low-pass filter with a $2 \mathrm{kHz}$ cutoff and sampled at $10 \mathrm{kHz}$ at room temperature $\left(25^{\circ} \mathrm{C}\right)$. A post-acquisition Gaussian filter of $200 \mathrm{~Hz}$ was applied. Error bars represent the standard deviation between independent experiments $(N=3)$

Simultaneous detection of glucose and asparagine in sweat. The residual current of the open configuration of SBD1 $\left(I_{\text {res } \%}=67.3\right.$ $\pm 0.3 \%$ ) was markedly different than the open protein configuration of GBP $\left(I_{\text {res } \%}=69.1 \pm 0.2 \%\right)$. Thus, when GBP $(44 \mathrm{nM})$ and SBD1 (120 nM) were simultaneously present in the cis solution, blockades corresponding to each protein adaptor could be readily distinguished (Fig. 3), allowing the simultaneous detection of glucose and asparagine from a sweat sample (Fig. 3).

\section{Discussion}

Portable devices for home diagnostics are sought after because they would reduce the cost of care and increase life expectancy by detecting diseases at an early stage. Here we showed that ClyA nanopores equipped with internal protein adapters can accurately measure the concentration of small metabolites by directly sampling tiny volumes of biological samples with no sample preparation. Such protein adapters are ideal biosensors, because they evolved over billion years to recognise biologically relevant molecules with high selectivity and sensitivity. Integration of ClyA nanopores with a panel of protein adapters in existing commercial portable devices would provide an off-the-shelf lowcost device for home diagnostics. The single-molecule nature of the sensor provides advantages over ensemble technologies, notably the ability of sampling of multiple analytes 

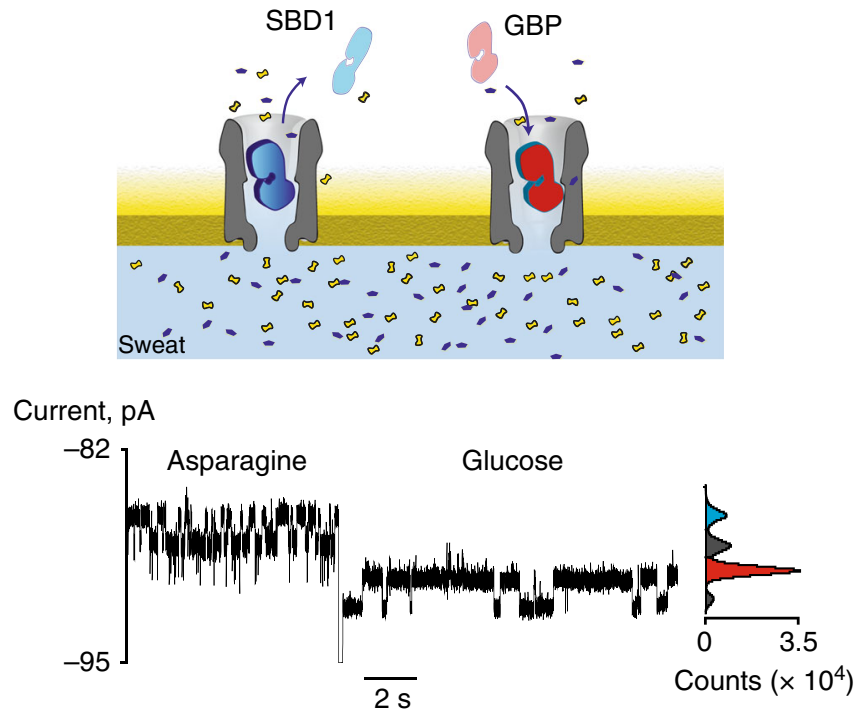

Fig. 3 Multiplexed detection of glucose and asparagine from sweat. Schematic representation (above) and current blockades with its all-point current histogram (below) of two consecutive events showing the entry of SBD1 and GBP into a ClyA nanopore, respectively, under $-90 \mathrm{mV}$ applied transmembrane potential. The trans solution contained $5 \mu \mathrm{L}$ of sweat constituting a 100-fold dilution. The solution used for the electrical recordings contained $150 \mathrm{mM} \mathrm{NaCl}$ and $15 \mathrm{mM}$ Tris- $\mathrm{HCl}$ set to $\mathrm{pH} 7.5$. Current traces were collected applying a Bessel low-pass filter with a $2 \mathrm{kHz}$ cutoff and sampled at $10 \mathrm{kHz}$ at room temperature $\left(25^{\circ} \mathrm{C}\right)$. A postacquisition Gaussian filter of $200 \mathrm{~Hz}$ was applied

simultaneously without the need of calibration for signal drift. Thus, nanopores equipped with internal protein adapters are ideal next-generation biosensors for miniaturised, non-invasive diagnostic, and wearable devices.

\section{Methods}

Chemicals, DNA, enzymes, and lipids. Unless otherwise specified all chemicals were bought from Sigma-Aldrich. DNA was purchased from Integrated DNA Technologies (IDT), enzymes from Thermo Fisher Scientific, and lipids from Avanti Polar Lipids.

GBP cloning. To allow cloning, a NcoI site (CCATGG) was introduced into the sequence of the wild-type GBP from Escherichia coli at the beginning of the gene ( $5^{\prime}$-end, beginning of the leader peptide). To maintain the open-reading frame, two extra bases were inserted after the NcoI site, resulting in an additional alanine residue after the starting methionine. For purification, a $\mathrm{His}_{6}$-tag was attached at the C-terminus of GBP, via a flexible glycine-serine-serine linker and the openreading frame was terminated by two consecutive stop codons, followed by a Hind III restriction site ( $3^{\prime}$-end). The attachment of the $\mathrm{His}_{6}$-tag was carried out in two consecutive PCR reactions. During the first PCR reaction, the GBP gene was amplified directly from a single BL21 (DE3) E. coli (Lucigen) colony using Phire Hot Start II DNA polymerase, $0.4 \mu \mathrm{M}$ dNTPs, $6 \mu \mathrm{M}$ of MS-Glu_F (TATATATC CATGGCTaataagaaggtgataaccetgtctgctgtg), and MS-Glu_r1 (gatggtgatgGCTGCT GCCtttcttgctgaattcagccaggttgtctttatctacgcc) primers in a $50 \mu \mathrm{L}$ reaction volume. The PCR reaction cycling protocol was as follows: pre-incubation step at $98^{\circ} \mathrm{C}$ for $30 \mathrm{~s}$ and then 30 cycles of denaturation at $98^{\circ} \mathrm{C}$ for $5 \mathrm{~s}$ and extension at $72^{\circ} \mathrm{C}$ for 1 min. The amplified product was purified using QIAquick PCR Purification Kit (Qiagen) and served as a template for the second PCR reaction, which used $\sim 100$ ng of the purified PCR product amplified by Phire Hot Start II DNA polymerase using $0.4 \mu \mathrm{M}$ dNTPs, $6 \mu \mathrm{M}$ of MS-Glu_F, and MS-Glu_r2 (atatata-

taagctttcattaatggtGATGGTGATGGCTGCTGCCTTTCTTGCTGAATTC) primers in $300 \mu \mathrm{L}$ volume. The cycling protocol was the same as in the previous step. The resulting PCR product encoding for the $\mathrm{His}_{6}$-tagged GBP gene was purified with QIAquick PCR Purification Kit (Qiagen) and digested with NcoI and HindIII (FastDigest, Fermentas). The gel purified insert (QIAquick Gel Extraction Kit, Qiagen) was cloned under control of the T7 promoter into the pT7-SC1 expression plasmid $^{29}$ using sticky-end ligation (T4 ligase, Fermentas) via NcoI $\left(5^{\prime}\right)$ and HindIII $\left(3^{\prime}\right)$ sites. Ligation mixture of $0.6 \mu \mathrm{L}$ was transformed into $50 \mu \mathrm{L}$ of E. cloni ${ }^{\circledast} 10 \mathrm{G}$ cells (Lucigen) by electroporation. The transformed bacteria were grown overnight at $37^{\circ} \mathrm{C}$ on ampicillin-containing $(100 \mu \mathrm{g} / \mathrm{mL}) \mathrm{LB}$ agar plates. The identity of the clones was confirmed by sequencing.

Protein sequence of GBP. MANKKVITLSAVMASMLFGAAAHAADTRIGVTIY KYDDNFMSVVRKAIEQDAKAAPDVQLLMNDSQNDQSKQNDQIDVLLAKGV KALAINLVDPAAAGTVIEKARGQNVPVVFFNKEPSRKALDSYDKAYYVG TDSKESGIIQGDLIAKHWAANQGWDLNKDGQIQFVLLKGEPGHPDAEART TYVIKELNDKGIKTEQLQLDTAMWDTAQAKDKMDAWLSGPNANKIEVV IANNDAMAMGAVEALKAHNKSSIPVFGVDALPEALALVKSGALAGT VLNDANNQAKATFDLAKNLADGKGAADGTNWKIDNKVVRV PYVGVDKDNLAEFSKKGSSHHHHH

DNA sequence of GBP. CCATGGCTAATAAGAAGGTGATAACCCTGTCTGC TGTGATGGCCAGCATGTTATTCGGTGCCGCTGCACACGCTGCTGATAC TCGCATTGGTGTAACAATCTATAAGTACGACGATAACTTTATGTCTGT AGTGCGCAAGGCTATTGAGCAAGATGCGAAAGCCGCGCCAGATGTTCA GCTGCTGATGAATGATTCTCAGAATGACCAGTCCAAGCAGAACGATCA GATCGACGTATTGCTGGCGAAAGGGGTGAAGGCACTGGCAATCAACCT GGTTGACCCGGCAGCTGCGGGTACGGTGATTGAGAAAGCGCGTGGGC AAAACGTGCCGGTGGTTTTCTTCAACAAAGAACCGTCTCGTAAGG CGCTGGATAGCTACGACAAAGCCTACTACGTTGGCACTGACTCCAAA GAGTCCGGCATTATTCAAGGCGATTTGATTGCTAAACACTGGGCG GCGAATCAGGGTTGGGATCTGAACAAAGACGGTCAGATTCAGTTCG TACTGCTGAAAGGTGAACCGGGCCATCCGGATGCAGAAGCACGTACCAC TTACGTGATTAAAGAATTGAACGATAAAGGCATCAAAACTGAACAGTTA CAGTTAGATACCGCAATGTGGGACACCGCTCAGGCGAAAGATAAGATG GACGCCTGGCTGTCTGGCCCGAACGCCAACAAAATCGAAGTGGTTAT CGCCAACAACGATGCGATGGCAATGGGCGCGGTTGAAGCGCTG AAAGCACACAACAAGTCCAGCATTCCGGTGTTTGGCGTCGATG CGCTGCCAGAAGCGCTGGCGCTGGTGAAATCCGGTGCACTGGC GGGCACCGTACTGAACGATGCTAACAACCAGGCGAAAGCGACCT TTGATCTGGCGAAAAACCTGGCCGATGGTAAAGGTGCGGCTGATGG CACCAACTGGAAAATCGACAACAAAGTGGTCCGCGTACCTTATG TTGGCGTAGATAAAGACAACCTGGCTGAATTCAGCAAGAAAGG CAGCAGCCATCACCATCACCATTAATGAAAGCTT

Construction GBP-A213R mutant. The GBP-A213R was constructed using the QuickChange protocol for site-directed mutagenesis ${ }^{30}$ on a circular plasmid template DNA (pT7-SC1 containing GBP gene) with Phire ${ }^{\varpi}$ Hot Start Polymerase (Finnzymes) by using two complementary primers. Six $\mu \mathrm{M}$ forward (GGTTATCGCCAACAACGATAGGATGGCA) and $6 \mu \mathrm{M}$ reverse primer (GCGCCCATTGCCATCCTATCGTTGTT) containing a codon encoding the mutation were used in $50 \mu \mathrm{L}$ final volume to amplify the gene (pre-incubation at $98^{\circ} \mathrm{C}$ for $30 \mathrm{~s}$, then 30 cycles of: denaturation at $98^{\circ} \mathrm{C}$ for $5 \mathrm{~s}$, extension at $72{ }^{\circ} \mathrm{C}$ for $1.5 \mathrm{~min}$ ). The PCR product was incubated with DpnI (1 FDU) for $1 \mathrm{~h}$ at $37^{\circ} \mathrm{C}$ to digest the plasmid template. The remaining DpnI enzyme was deactivated at $55^{\circ} \mathrm{C}$. The mixture was then incorporated into E. cloni ${ }^{\oplus} 10 \mathrm{G}$ cells (Lucigen) by electroporation. Transformants containing the plasmid were grown overnight at $37^{\circ} \mathrm{C}$ on LB agar plates supplemented with $100 \mu \mathrm{g} / \mathrm{mL}$ ampicillin and $1 \%$ glucose. Single colonies were picked and inoculated into $10 \mathrm{~mL} \mathrm{LB}$ medium supplemented with $100 \mathrm{mg} / \mathrm{L}$ of ampicillin for plasmid DNA preparation. The presence of the mutation was confirmed by sequencing of the plasmid.

Purification of SBD1. Substrate-binding domains carrying a histidine-tag were expressed in E. coli strain MC1061 and purified as described previously. ${ }^{28}$ The cell lysate was mixed with $50 \mathrm{mM}$ potassium phosphate, $\mathrm{pH} 8.0,200 \mathrm{mM} \mathrm{KCl}, 20 \%$ glycerol (buffer A) supplied with $20 \mathrm{mM}$ imidazole and incubated with $\mathrm{Ni}^{2}$ ${ }^{+}$-sepharose (GE Healthcare) resin for $1 \mathrm{~h}$ at $4^{\circ} \mathrm{C}$. Next, the resin was washed with 20 column volumes of buffer A supplied with $50 \mathrm{mM}$ imidazole, followed by protein elution with $500 \mathrm{mM}$ imidazole in buffer A. To prevent protein aggregation, $5 \mathrm{mM}$ EDTA was added immediately after elution. Afterwards the histidinetag was cleaved off by adding $2.5 \% \mathrm{w} / \mathrm{w}$ His-TEV $(\mathrm{S} 219 \mathrm{~V})^{31}$ and dialyzing overnight against $50 \mathrm{mM}$ Tris- $\mathrm{Cl}, 0.5 \mathrm{mM}$ DTT, $0.5 \mathrm{mM}$ EDTA, $\mathrm{pH} 8.0$ to remove imidazole. The histidine-tagged tobacco etch virus (TEV) protease and residual uncut protein were removed by loading it on a small $0.5 \mathrm{~mL} \mathrm{Ni}^{2+}$-sepharose column collecting the flow-through. Next, proteins were further purified with sizeexclusion chromatography on a Superdex-200 column (GE Healthcare) equilibrated with $50 \mathrm{mM}$ Tris- $\mathrm{HCl}, 200 \mathrm{mM} \mathrm{NaCl}, \mathrm{pH}$ 7.5. Peak fractions were collected in aliquots and were stored at $-80^{\circ} \mathrm{C}$ after flash-freezing in liquid nitrogen.

Purification of ClyA-AS. E. cloni ${ }^{\boxplus}$ EXPRESS BL21 (DE3) cells were transformed with the pT7-SC1 plasmid containing the ClyA-AS gene. ClyA-AS contains eight mutations relative to the S. Typhi ClyA-WT: C87A, L99Q, E103G, F166Y, I203V, C285S, K294R, and H307Y. Transformants were selected after overnight growth at $37^{\circ} \mathrm{C}$ on LB agar plates supplemented with $100 \mathrm{mg} / \mathrm{L}$ ampicillin and $1 \%$ glucose. The resulting colonies were inoculated into $400 \mathrm{~mL} 2 \mathrm{xYT}$ medium containing 100 $\mathrm{mg} / \mathrm{L}$ of ampicillin. The cells were grown at $37^{\circ} \mathrm{C}(200$ r.p.m. shaking $)$ until an $\mathrm{OD}_{600}$ of $\sim 0.8$ was reached. The expression of ClyA-AS was induced by addition of $0.5 \mathrm{mM}$ IPTG. The cell cultures were further grown overnight at $25^{\circ} \mathrm{C}$. The next 
day, the bacteria were harvested by centrifugation at $6000 \times \mathrm{g}$ at $4{ }^{\circ} \mathrm{C}$ for $25 \mathrm{~min}$ and the pellets were stored at $-80^{\circ} \mathrm{C}$. The pellets containing monomeric ClyA-AS were thawed and solubilized with $20 \mathrm{~mL}$ lysis buffer $(150 \mathrm{mM} \mathrm{NaCl}, 15 \mathrm{mM}$ Tris-HCl, $\mathrm{pH} 7.5,1 \mathrm{mM} \mathrm{MgCl} 2,0.2$ units per mL DNaseI, $10 \mathrm{mM}$ imidazole, $10 \mu \mathrm{g} / \mathrm{mL}$ lysozyme) and incubated for $20 \mathrm{~min}$ at $37^{\circ} \mathrm{C}$. The bacteria were then lysed by sonication (Branson). The lysate was subsequently centrifuged at $6000 \times g$ at $4{ }^{\circ} \mathrm{C}$ for $20 \mathrm{~min}$ and the cellular debris discarded. The supernatant was mixed with $150 \mu \mathrm{L}$ of Ni-NTA resin (Qiagen) in wash buffer $(10 \mathrm{mM}$ imidazole, $150 \mathrm{mM} \mathrm{NaCl}, 15$ $\mathrm{mM}$ Tris-HCl, $\mathrm{pH}$ 7.5). After $1 \mathrm{~h}$, the resin was loaded into a column (Micro Bio Spin, Bio-Rad) and washed with $\sim 5 \mathrm{~mL}$ of the wash buffer. The protein was eluted with $\sim 100 \mu \mathrm{L}$ elution buffer $(300 \mathrm{mM}$ imidazole, $150 \mathrm{mM} \mathrm{NaCl}, 15 \mathrm{mM}$ Tris-HCl, $\mathrm{pH}$ 7.5). Type I ClyA-AS oligomers were obtained by incubation of ClyA-AS monomers with $0.2 \% \beta$-dodecylmaltoside (DDM) for $30 \mathrm{~min}$ at $37^{\circ} \mathrm{C}$. The oligomers were separated from monomers by blue native polyacrylamide gel electrophoresis (BN-PAGE, Bio-Rad) using $4-20 \%$ polyacrylamide gels ${ }^{20}$. The band corresponding to type I ClyA-AS were cut out from the gel and was extracted in $150 \mathrm{mM} \mathrm{NaCl}, 15 \mathrm{mM}$ Tris- $\mathrm{HCl}, \mathrm{pH} 7.5$ supplemented with $0.2 \%$ DDM and 10 mM EDTA.

Purification of GBP. E. cloni ${ }^{\circledR}$ EXPRESS BL21 (DE3) cells were transformed with the pT7-SC1 plasmid containing the $\mathrm{His}_{6}$-tagged GBP gene. Transformants were selected after overnight growth at $37^{\circ} \mathrm{C}$ on LB agar plates supplemented with 100 $\mathrm{mg} / \mathrm{L}$ ampicillin and $1 \%$ glucose. The resulting colonies were inoculated into 400 $\mathrm{mL} 2$ XYT medium containing $100 \mathrm{mg} / \mathrm{L}$ of ampicillin. The cells were grown at $37^{\circ}$ C (200 r.p.m. shaking) until an $\mathrm{OD}_{600}$ of $\sim 0.8$ was reached. The expression of GBP was induced by addition of $0.5 \mathrm{mM}$ IPTG. The cell cultures were further grown overnight at $25^{\circ} \mathrm{C}$. The next day, the bacteria were harvested by centrifugation at $6000 \times g$ at $4{ }^{\circ} \mathrm{C}$ for $25 \mathrm{~min}$. The protein was purified directly afterwards by osmotic shock. The pellets were thoroughly dissolved in $20 \%$ ice-cold sucrose solution and incubated, while shaking at $4{ }^{\circ} \mathrm{C}$ for $10 \mathrm{~min}$. The cell suspension was centrifuged at $6000 \times g$ at $4^{\circ} \mathrm{C}$ for $10 \mathrm{~min}$ and cells collected. The cells were re-suspended in $4 \times$ volume of ice-cold MilliQ water supplemented with $5 \mathrm{mM} \mathrm{MgCl}_{2}$ and incubated, while shaking, on ice for $20 \mathrm{~min}$. The cells collected again by centrifugation at $6000 \times g$ at $4^{\circ} \mathrm{C}$ for $20 \mathrm{~min}$. The supernatant was mixed with $150 \mu \mathrm{L}$ of Ni-NTA resin (Qiagen) in wash buffer (10 $\mathrm{mM}$ imidazole, $150 \mathrm{mM} \mathrm{NaCl}, 15 \mathrm{mM}$ Tris- $\mathrm{HCl}$, $\mathrm{pH}$ 7.5). After $1 \mathrm{~h}$, the resin was loaded into a column (Micro Bio Spin, Bio-Rad) and washed with $\sim 5 \mathrm{~mL}$ of the wash buffer. The protein was eluted with $\sim 500 \mu \mathrm{L}$ elution buffer ( $300 \mathrm{mM}$ imidazole, $150 \mathrm{mM} \mathrm{NaCl}, 15 \mathrm{mM}$ Tris-HCl, $\mathrm{pH}$ 7.5). The protein $(500 \mu \mathrm{L})$ was then dialysed exhaustively overnight against dialysis buffer (total volume of $1500 \mathrm{~mL} ; 5 \mathrm{mM}$ EDTA, $150 \mathrm{mM} \mathrm{NaCl}, 15 \mathrm{mM}$ Tris-HCl, $\mathrm{pH}$ 7.5), whereby the dialysis buffer was replaced for fresh buffer at least three times. Protein concentration was measured by using the Nanodrop $2000 \mathrm{C}$ at $280 \mathrm{~nm}$ (Thermo Fisher Scientific). Protein was stored with $10 \%$ glycerol in $-80^{\circ} \mathrm{C}$ after flashfreezing until further use.

Electrical recordings in planar lipid bilayers. The setup consisted of two chambers separated by a $25 \mu \mathrm{m}$ thick polytetrafluoroethylene film (Goodfellow Cambridge Limited) containing an aperture of approximately $100 \mu \mathrm{m}$ in diameter. The aperture was pre-treated with a solution of hexadecane $[10 \%(\mathrm{v} / \mathrm{v})$ in pentane] to leave a hydrophobic coating to support bilayer formation. 1,2-diphytanoyl-snglycero-3-phosphocholine (DPhPC) in pentane $(10 \mathrm{mg} / \mathrm{mL})$ was then added to the buffer $(500 \mu \mathrm{L}, 150 \mathrm{mM} \mathrm{NaCl}, 15 \mathrm{mM}$ Tris-HCl, pH 7.5) present in both compartments. After evaporation of the pentane, a lipid monolayer at the air-water interface can self-assemble spontaneously by lowering and raising the buffer across the aperture. 0.01-0.1 ng of purified ClyA-AS were added to the grounded cis compartment. The reconstitution of single nanopores was monitored electrically by applying a $-35 \mathrm{mV}$ bias. ClyA-AS nanopores display a higher open pore current at positive than at negative applied potentials, which provides a useful tool to determine the orientation of the pore. After insertion of a single pore, GBP or SBD1 were added to the cis compartment. This was followed by addition of a glucose or asparagine sample or bodily fluid sample in the trans compartment.

Data recordings and analysis. All traces were routinely recorded at $-90 \mathrm{mV}$ or $-60 \mathrm{mV}$ applied transmembrane potential by using an Axopatch $200 \mathrm{~B}$ patch clamp amplifier (Axon Instruments) and digitised with a DigiData $1440 \mathrm{~A} / \mathrm{D}$ converter (Axon Instruments). The data was sampled by applying a $2 \mathrm{kHz}$ low-pass Bessel filter and at a frequency of $10 \mathrm{kHz}$. Obtained traces were filtered digitally with a Gaussian low-pass filter with a $100 \mathrm{~Hz}$ cutoff. Data were recorded by using the Clampex 10.4 software (Molecular Devices) and the subsequent analysis was carried out with the Clampfit software (Molecular Devices).

Analysis of current traces. Current transitions between the open and closed levels were analysed using the "single-channel search" function using Clampfit software (Molecular Devices). Events shorter than $2 \mathrm{~ms}$ were ignored. The mean dwell times for the ligand-induced closing $\left(\tau_{\text {on }}\right)$ and ligand-independent opening $\left(\tau_{\text {off }}\right)$ of GBP were obtained from single exponential fits to histograms of the inter-event times and dwell times, respectively. The mean lifetime of the open state $\left(\tau_{\text {on }}\right)$ was converted to the on-rate constants by using $k_{\text {on }}=1 / \tau_{\text {on }}$ [glucose], where [glucose] is the concentration of glucose added to the trans side. The $\tau_{\text {off }}$ was converted to the off-rate by using $k_{\text {off }}=1 / \tau_{\text {off }}$. The equilibrium binding constant was calculated by plotting the fraction of closed configuration against the concentration of substrate and the data fitted to a Hill function (Fig. 1c). The $K_{\mathrm{d}}^{\mathrm{app}}$ is the concentration of substrate at $50 \%$ signal saturation.

Calibration curve for GBP binding to glucose. Values of the relative open/closed population were obtained from Gaussian fittings to all-point current histograms (Fig. 1b). In a typical experiment, an all-point histogram was created from individual current blockade longer than $\sim 5 \mathrm{~s}$. Gaussian fittings were then used to measure the area under the curve corresponding to the open and closed configuration for each protein blockade. Then, all areas were added together to calculate the overall percentage of open and closed configuration. Since the open and closed configurations were still observed in the absence of glucose or at saturated concentration of glucose (e.g., $50 \mu \mathrm{M}$, Supplementary Fig. 1b), the open/closed population was normalised using the following equation:

$$
x_{\text {norm }}=\frac{x-x_{\min }}{0.94-x_{\min }},
$$

where $x_{\text {norm }}$ is the normalised value, $x_{\min }$ is the fraction of the apo-protein in the closed configuration measured during each experiment before addition of glucose, and 0.94 is the fraction of closed configuration at saturating glucose concentrations. The fraction of closed configuration was then plotted against the concentration of substrate and the data fitted to a Hill function (Fig. 1c). This graph was used as a calibration curve to determine the ligand concentration in the bodily fluids.

Commercial glucose tests. A glucose assay kit (HK) from Sigma-Aldrich and a commercial glucose metre (Accu-chek ${ }^{\star}$ Performa, Roche, Switzerland) were used to determine the glucose in the bodily fluids. The solution of the glucose assay kit contains a hexokinase enzyme that uses adenosine triphosphate (ATP) as a cofactor to phosphorylate glucose. The glucose-6-phosphate (G6P) is then oxidised to 6-phospho-gluconate in the presence of oxidised nicotinamide adenine dinucleotide (NAD) in a reaction catalysed by glucose-6-phosphate dehydrogenase (G6PD) The generation of NADH was spectrophotometrically observed by measuring absorbance at $340 \mathrm{~nm}$. Under these conditions, the final concentration of produced NADH corresponds to the glucose concentration in the sample. In order to perform the experiment, a dilution of bodily fluid was added to the reaction mixture The reaction was incubated for $15 \mathrm{~min}$ at room temperature and then the absorbance was measured at $340 \mathrm{~nm}\left(\varepsilon=6220 \mathrm{M}^{-1} \mathrm{~cm}^{-1}\right)$. For each sample, three different dilutions were measured. The commercial glucose metre Accu-Chek ${ }^{\circ}$ uses a mutant variant of quinoprotein glucose dehydrogenase and has a measurement range between 0.6 and $33.3 \mathrm{mmol} / \mathrm{L}^{32}$. The Accu-Chek ${ }^{\circledR}$ is considered to be a reliable device for monitoring glucose levels in blood from finger pricks ${ }^{33}$.

Sample collection. The bodily fluids were collected from two healthy volunteers who were fasting for at least $1 \mathrm{~h}$ (woman in her 20's and men in his 30's). The blood samples were collected shortly before the experiments to minimise ex vivo artefactual changes. Sweat, urine, and saliva samples were either freshly collected before, or stored at $-20^{\circ} \mathrm{C}$, before the start of experiments. The solutions were kept on ice for the duration of an experiment. The blood samples were diluted 500-folds and the urine sample 10 -folds with buffer $(150 \mathrm{mM} \mathrm{NaCl}, 15 \mathrm{mM}$ Tris- $\mathrm{HCl}, \mathrm{pH}$ 7.5) before adding 5 and $2 \mu \mathrm{L}$, respectively, to the trans compartment.

Measurement time. The concentration of analytes in blood was measured by calculating the relative fraction of the open vs. closed configuration from Gaussian fittings to all-point current histograms over a certain integration time (Fig. 1b). For asparagine, the integration time was $\sim 30 \mathrm{~s}$, and for glucose $\sim 10 \mathrm{~min}$. We tested smaller integration times (Supplementary Fig. 7) and found that at least $5 \mathrm{~min}$ are necessary to obtain accurate measurements for glucose and about $10 \mathrm{~s}$ for asparagine. The difference between the two analyte lies in the different affinities of the proteins for their ligands. Glucose binds relatively strongly to $\mathrm{GBP}\left(k_{\text {opening }}=0.75\right.$ $\pm 0.12 \mathrm{~s}^{-1}, 500 \mathrm{nM}$ glucose), compared to the binding of asparagine to SBD1 $\left(k_{\text {opening }}=8.6 \pm 0.1 \mathrm{~s}^{-1}, 800 \mathrm{nM}\right.$ asparagine), thus longer integration times are required for accurate reading. It follows that (GBP-A213R), which shows reduced affinity for glucose $\left(k_{\text {opening }}=121.0 \pm 6.9 \mathrm{~s}^{-1}\right.$ at $20 \mu \mathrm{M}$ glucose, Supplementary Fig. 2), will provide faster reading times, with lower sensitivity, but wider detection range.

Bilayer stability. All measurements were made by adding small volumes of biological samples to an electrophysiology chamber $(500 \mu \mathrm{L})$. We did not observe a particular instability of the bilayer (Supplementary Figs. 3 and 4), except when lower than 1:50 or 1:30 dilutions of serum or saliva were used (Supplementary Fig. 4). Typically, with a 1:33 dilution of serum the lipid bilayer was stable for $9.4 \pm$ $9.2 \mathrm{~min}$ (the error is $\mathrm{SD}, N=10$ ). 
Measurement of asparagine using high performance liquid chromatography

(HPLC). Urine and sweat samples were analysed without any pretreatment directly after the addition of $50 \mu \mathrm{L}$ standard additions to $50 \mu \mathrm{L}$ sample while phosphate buffered saline was used as a blank.

Proteins in $150 \mu \mathrm{L}$ saliva and $60 \mu \mathrm{L}$ serum samples were precipitated by incubation with $8 \%$ trichloroaceticacid (CASnr 76-03-9) for $5 \mathrm{~min}$ at $60^{\circ} \mathrm{C}$ and 450 r.p.m. using a thermomixer (comfort, Eppendorf, Hamburg, Germany) followed by cooling on ice for $30 \mathrm{~min}$ and centrifugation for $10 \mathrm{~min}$ at $20,000 \times g$ and $4{ }^{\circ} \mathrm{C}$. A fixed volume of the supernatant was taken out using a pipette not taking any precipitate and neutralised with a four times larger volume of saturated sodium borate (CASnr 1303-96-4). After mixing, a concentrator (SpeedVac 5301, Eppendorf, Hamburg, Germany) was used to evaporate all liquids by vacuum evaporation at $60^{\circ} \mathrm{C}$ and $240 \times g$, and the sample was reconstituted in an identical volume of water as the former fixed amount of supernatant. A final 5-min-long centrifugation at 20,000 $\times g$ step was performed and the supernatant was stored at $4{ }^{\circ} \mathrm{C}$ before analysis.

HPLC quantification of asparagine was performed on an Agilent 1100 HPLC binary system (Agilent, Santa Clara, USA) equipped with an 1100 Fluorescence detector (FLD) and a Gemini C18 column $(2 \times 250 \mathrm{~mm}, 5 \mu \mathrm{m}$, Phenomenex, Torrance, USA) while a $40 \mathrm{mM}$ sodium phosphate buffer, $\mathrm{pH} 7.8$ (solvent A) and a mixture of 45:45:10 acetonitrile/methanol/water (solvent B) were used. The fluorogenic labelling of amino acids was performed automatically by the auto sampler of the HPLC machine using a mixture of $10 \mathrm{mg}$ o-phtalaldehyde (OPA, CASnr 643-79-8) and $10 \mu \mathrm{L}$ 3-mercaptapropionic acid (3MPA, CASnr 107-96-0) in water as reagent. The auto sampler mixed $0.5 \mu \mathrm{L}$ sample with $1.3 \mu \mathrm{L} 0.4 \mathrm{~N}$ borate buffer, $\mathrm{pH} 10.2$ followed by $0.5 \mu \mathrm{L}$ reagent and $7.6 \mu \mathrm{L}$ water right before each injection. After the sample injection, amino acids were separated by applying solvent $\mathrm{B}$ at $1.5 \% / \mathrm{min}$ increase for $25 \mathrm{~min}$ using a flow rate of $250 \mu \mathrm{L} / \mathrm{min}$ (the initial solvent B concentration was 5\%). Thereafter, the column was cleaned for 5 min at $66 \%$ solvent B followed by another $12 \mathrm{~min}$ at $100 \%$ solvent $\mathrm{B}$. Then the column was equilibrated back to $5 \%$ solvent B. Fluorescence was measured at an excitation wavelength of $340 \mathrm{~nm}$ and an emission wavelength of $450 \mathrm{~nm}$.

Quantifications were performed using the method of standard addition at three different concentrations of analyte $(10,50$, and $200 \mu \mathrm{M})$. The concentration of the analyte was obtained by measuring the peak areas, while each sample was analysed in triplicate. Citrulline was added as internal standard at $200 \mu \mathrm{M}$ to correct for saliva and serum sample handling volume differences next to control run-to-run retention time shifts regarding all samples.

\section{Data availability}

The datasets generated during and/or analysed during the current study are available from the corresponding author on reasonable request.

Received: 17 July 2018 Accepted: 30 August 2018

Published online: 05 October 2018

\section{References}

1. Rocchitta, G. et al. Enzyme biosensors for biomedical applications: strategies for safeguarding analytical performances in biological fluids. Sensors 16, 780 (2016).

2. Clark, L. C. Jr. \& Lyons, C. Electrode systems for continuous monitoring in cardiovascular surgery. Ann. N. Y. Acad. Sci. 102, 29-45 (1962).

3. D’Orazio, P. Biosensors in clinical chemistry. Clin. Chim. Acta 334, 41-69 (2003).

4. Zhu, C., Yang, G., Li, H., Du, D. \& Lin, Y. Electrochemical sensors and biosensors based on nanomaterials and nanostructures. Anal. Chem. 87, 230-249 (2015)

5. Grieshaber, D., MacKenzie, R., Vörös, J. \& Reimhult, E. Electrochemical biosensors - sensor principles and architectures. Sensors 8, 1400-1458 (2008).

6. Bayley, H. Nanopore sequencing: from imagination to reality. Clin. Chem. 61 , 25-31 (2015)

7. Huang, G., Willems, K., Soskine, M., Wloka, C. \& Maglia, G. Electro-osmotic capture and ionic discrimination of peptide and protein biomarkers with FraC nanopores. Nat. Commun. 8, 935 (2017)

8. Chavis, A. E. et al. Single molecule nanopore spectrometry for peptide detection. ACS Sens. 2, 1319-1328 (2017).

9. Holmes, E. et al. Human metabolic phenotype diversity and its association with diet and blood pressure. Nature 453, 396-400 (2008).

10. Gu, L.-Q., Braha, O., Conlan, S., Cheley, S. \& Bayley, H. Stochastic sensing of organic analytes by a pore-forming protein containing a molecular adapter. Nature 398, 686-690 (1999).

11. Braha, O., Webb, J., Gu, L.-Q., Kim, K. \& Bayley, H. Carriers versus adapters in stochastic sensing. Chemphyschem 6, 889-892 (2005).
12. Sanchez-Quesada, J., Ghadiri, M. R., Bayley, H. \& Braha, O. Cyclic peptides as molecular adapters for a pore-forming protein. J. Am. Chem. Soc. 122, 11757-11766 (2000)

13. Soskine, M., Biesemans, A. \& Maglia, G. Single-molecule analyte recognition with ClyA nanopores equipped with internal protein adaptors. J. Am. Chem. Soc. 137, 5793-5797 (2015).

14. Van Meervelt, V., Soskine, M. \& Maglia, G. Detection of two isomeric binding configurations in a protein-aptamer complex with a biological nanopore. ACS Nano 8, 12826-12835 (2014).

15. Soskine, M. et al. An engineered ClyA nanopore detects folded target proteins by selective external association and pore entry. Nano Lett. 12, 4895-4900 (2012).

16. Van Meervelt, V. et al. Real-time conformational changes and controlled orientation of native proteins inside a protein nanoreactor. J. Am. Chem. Soc. 139, 18640-18646 (2017).

17. Ge, X., Tolosa, L., Simpson, J. \& Rao, G. Genetically engineered binding proteins as biosensors for fermentation and cell culture. Biotechnol. Bioeng. 84, 723-731 (2003)

18. Berntsson, R. P.-A., Smits, S. H. J., Schmitt, L., Slotboom, D.-J. \& Poolman, B. A structural classification of substrate-binding proteins. FEBS Lett. 584, 2606-2617 (2010).

19. Unione, L. et al. Unraveling the conformational landscape of ligand binding to glucose/galactose-binding protein by paramagnetic NMR and MD simulations. ACS Chem. Biol. 11, 2149-2157 (2016).

20. Soskine, M., Biesemans, A., De Maeyer, M. \& Maglia, G. Tuning the size and properties of ClyA nanopores assisted by directed evolution. J. Am. Chem. Soc 135, 13456-13463 (2013)

21. Miller, D. M., Olson, J. S. \& Quiocho, F. A. The mechanism of sugar binding to the periplasmic receptor for galactose chemotaxis and transport in Escherichia coli. J. Biol. Chem. 255, 2465-2471 (1980).

22. Zukin, R. S., Strange, P. G., Heavey, R. \& Koshland, D. E. Properties of the galactose binding protein of Salmonella typhimurium and Escherichia coli. Biochemistry 16, 381-386 (1977).

23. Miller, D. M., Olson, J. S., Pflugrath, J. W. \& Quiocho, F. A. Rates of ligand binding to periplasmic proteins involved in bacterial transport and chemotaxis. J. Biol. Chem. 258, 13665-13672 (1983).

24. Khan, F. \& Pickup, J. C. Near-infrared fluorescence glucose sensing based on glucose/galactose-binding protein coupled to 651-blue oxazine. Biochem. Biophys. Res. Commun. 438, 488-492 (2013).

25. Helassa, N. et al. A novel fluorescent sensor protein for detecting changes in airway surface liquid glucose concentration. Biochem. J. 464, 213-220 (2014).

26. Armengou, A. et al. L-arginine levels in blood as a marker of nitric oxidemediated brain damage in acute stroke: a clinical and experimental study. J. Cereb. Blood Flow Metab. 23, 978-984 (2003).

27. Jiménez-Jiménez, F. J. et al. Neurotransmitter amino acids in cerebrospinal fluid of patients with Alzheimer's disease. J. Neural Transm. 105, 269-277 (1998)

28. Gouridis, G. et al. Conformational dynamics in substrate-binding domains influences transport in the ABC importer GlnPQ. Nat. Struct. Mol. Biol. 22, 57-64 (2015)

29. Miles, G., Cheley, S., Braha, O. \& Bayley, H. The staphylococcal leukocidin bicomponent toxin forms large ionic channels. Biochemistry 40, 8514-8522 (2001).

30. Papworth, C., Bauer, J. C., Braman, J. \& DA., W. Site-directed mutagenesis in one day with $>80 \%$ efficiency. Strategies 9, 3 (1996).

31. Kapust, R. B. et al. Tobacco etch virus protease: mechanism of autolysis and rational design of stable mutants with wild-type catalytic proficiency. Protein Eng. 14, 993-1000 (2001).

32. Sutheran, H. L. \& Reynolds, T. Technical and clinical accuracy of three blood glucose meters: clinical impact assessment using error grid analysis and insulin sliding scales. J. Clin. Pathol. 69, 899-905 (2016).

33. Tack, C. et al. Accuracy evaluation of five blood glucose monitoring systems obtained from the pharmacy: a European multicenter study with 453 subjects. Diabetes Technol. Ther. 14, 330-337 (2012).

\section{Acknowledgements}

C.W. work was supported by Oxford Nanopore Technologies Ltd. and by the Netherlands Organisation for Scientific Research (NWO Veni, 722.017.010). The work of G.M. was funded by an ERC consolidator grant (SMEN\#260884). Gea K. SchuurmanWolters provided the SBD1 protein.

\section{Author contributions}

N.S.G. performed the experiments and analysed the data presented in the manuscript. M.S. cloned the DNA. J.H. performed HPLC measurements. M.S. and C.W. performed 
preliminary electrophysiological experiments. N.S.G., C.W., and G.M. wrote the manuscript with contributions from M.S. G.M. supervised the project.

\section{Additional information}

Supplementary Information accompanies this paper at https://doi.org/10.1038/s41467018-06534-1.

Competing interests: C.W. was partially sponsored by Oxford Nanopore Technologies Ltd. The remaining authors declare no other competing interests.

Reprints and permission information is available online at http://npg.nature.com/ reprintsandpermissions/

Publisher's note: Springer Nature remains neutral with regard to jurisdictional claims in published maps and institutional affiliations. (c) Open Access This article is licensed under a Creative Commons Attribution 4.0 International License, which permits use, sharing, adaptation, distribution and reproduction in any medium or format, as long as you give appropriate credit to the original author(s) and the source, provide a link to the Creative Commons license, and indicate if changes were made. The images or other third party material in this article are included in the article's Creative Commons license, unless indicated otherwise in a credit line to the material. If material is not included in the article's Creative Commons license and your intended use is not permitted by statutory regulation or exceeds the permitted use, you will need to obtain permission directly from the copyright holder. To view a copy of this license, visit http://creativecommons.org/ licenses/by/4.0/.

(C) The Author(s) 2018 